\title{
Natural Convection Flow in A Square Cavity with Temperature Dependent Heat Generation
}

\author{
Farjana Habiba \\ Department of Natural Science, Stamford University Bangladesh
}

\begin{abstract}
Unsteady natural convection laminar flows in a square cavity formed by insulated bottom and top walls, uniformly heated left wall and cooled right wall has been investigated. The governing equations are transformed into a non-dimensional form and the resulting partial differential equations are solved numerically applying upwind finite difference method together with Successive Over-Relaxation (SOR) scheme. The effect of the heat generation and the Rayleigh number on streamlines and isotherms as well as on the local rate of heat transfer in terms of the local Nusselt number and the average Nusselt number from the heated wall of the cavity are presented.
\end{abstract}

Keywords: Natural convection, Unsteady laminar flow, Heat generation, Square cavity

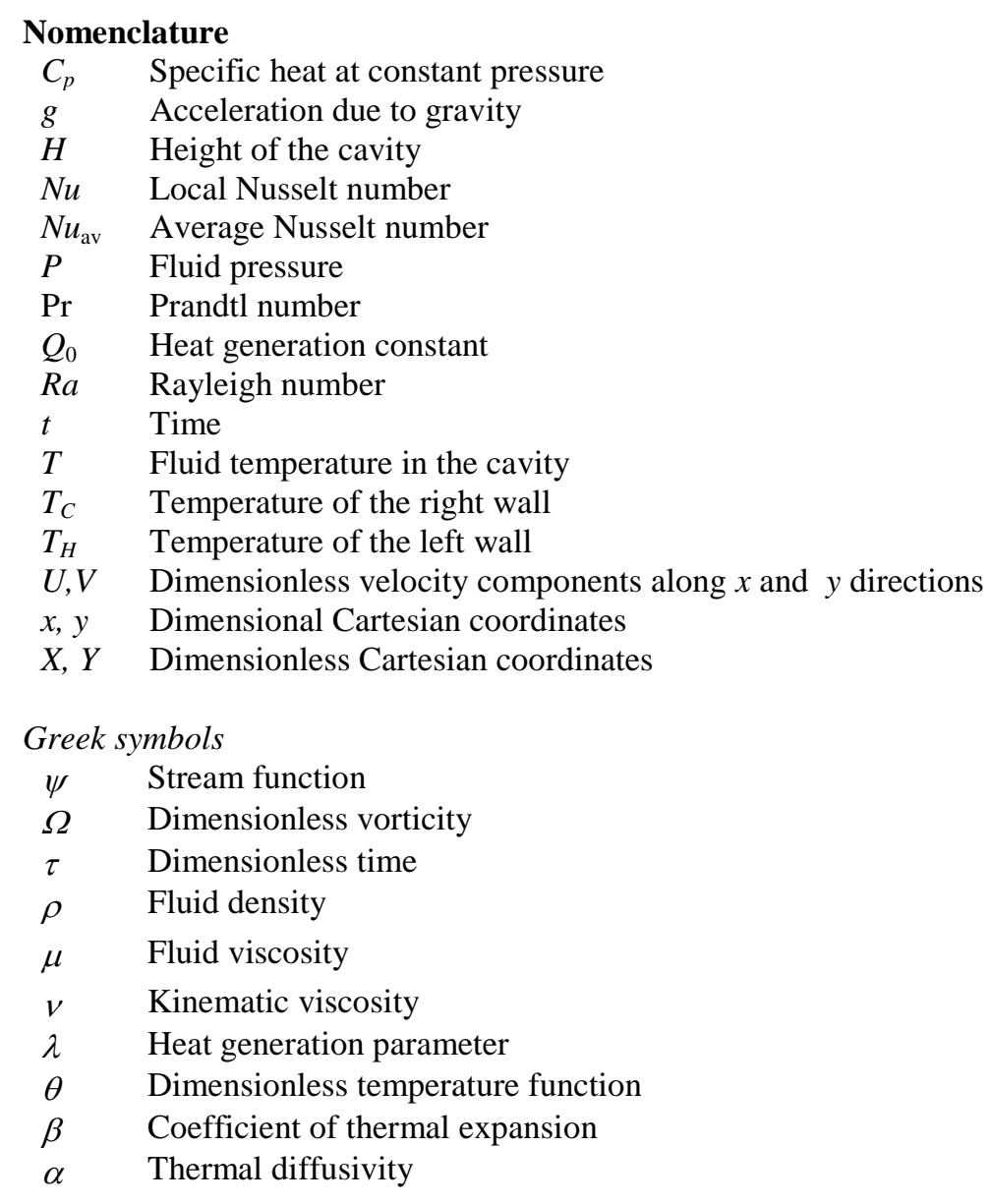

\section{Introduction}

Natural convection in enclosures has attracted considerable interest of investigators as the transport process in a fluid where the motion drives by the interactions of a difference in density with a gravitational field is very common in several engineering and environmental problems. Application of such analysis is essential for building design, that is, ventilation, solar energy systems, electronic equipment cooling, meteorology, geophysics, nuclear reactor systems, fire control and chemical. Therefore natural convection represents in several fields, where the heat to be dissipated is low enough and an attractive system in thermal control because of its low cost, reliability and simplicity in use. 
Ostrach [1] provided a comprehensive review article and extensive bibliography on natural convection in cavities up to 1988. de Vahl Davis [2] investigated the natural convection of air in a square cavity. The author assumed the top and bottom walls are adiabatic and left wall is uniformly heated, right wall is cooled. Valencia and Frederick [3] investigated the heat transfer in square cavities with partially active vertical walls. Selamet et al. [4] studied the laminar buoyancy driven flows in an enclosure. Natural convection heat transfer in rectangular cavities heated from below had been investigated by Hasnaoui et al. [5]. Sundstrom and Kimura [6] observed the phenomena on laminar free convection in inclined rectangular enclosure. November and Nansteel [7], Ganzarolli and Milanez [8], investigated the natural convection in rectangular enclosures heated form below and cooling from the sides. Natural convection in cavities heating from below and cooling from the side walls as well as heating from one side and cooling from ceiling has been studied by Aydin et al. [9-10], Sharif and Mohammad [11]. Sezai and Mohammad [12] studied the natural convection from a discrete heat source on the bottom of horizontal enclosure. Following de Vahl Davis [2], Dixit and Babu [13] have recently investigated natural convection of air in square cavity by using the lattice Boltzmann method.

A large number of physical phenomena involves with natural convection driven by heat generation. The study of heat generation in moving fluids is important in view of several physical problems such as those dealing with chemical reactions and those concerned with dissociating fluids. Possible heat generation effects may alter the temperature distribution and, therefore, the particle deposition rate. This may occur in such applications related to nuclear reactor cores, fire and combustion modelling, electronic chips and semiconductor wafers. In fact, the literature is replete with examples dealing with the heat transfer in laminar flow of viscous fluids. Barozzi and Corticelli [14] investigated the natural convection in cavities with internal heat source. Natural convection in vertical cavities with internal heat generation porous medium has been studied by Du and Bilgen [15]. Baytas [16] investigated the buoyancy-driven flow in an enclosure containing time periodic internal heat sources.

All of the above authors ignored the effect of temperature dependent heat generation due to molecular interaction within the cavities, winch is more important in point of practical applications. Vajravelu and Hadjinolaou [17] studied the heat transfer characteristics in a laminar boundary layer flow of a viscous fluid over a linearly stretching continuous surface with temperature dependent internal heat generation. Recently, Molla et al. [18-20] have investigated the natural convection flow with temperature dependent heat generation along a uniformly heated vertical wavy surface, horizontal circular cylinder and sphere.

In the present study, we are interested to investigate the unsteady natural convection laminar flow in a square cavity formed by bottom and top walls are insulated, left wall is uniformly heated and the right wall is cooled .The basic equations of motion are transformed into a non-dimensional form, which are solved numerically by using a upwind finite-difference scheme together with Successive Over Relaxation (SOR) scheme. The effect of the heat generation and the Rayleigh number on streamlines and isotherms, as well as on local the rate of heat transfer and the average rate of heat transfer from the heated wall of the cavity are presented by graphically. In the whole investigation the Prandtl number Pr has been chosen 1.0 without the comparison with the benchmark solutions given by de Vahl Davis [2].

\section{Formulation Of Problem}

Consider a square cavity of height $H$ filled with viscous incompressible fluid as shown in Fig. 1. The top and bottom walls are adiabatic where as the right wall is maintained at constant cold temperature and the left wall is isothermally heated. We also bring into account the effect of temperature-dependent heat generation as [14] in the flow region. The volumetric rate of heat generation $q^{\prime \prime \prime}\left[\mathrm{W} / \mathrm{m}^{3}\right]$, is assumed to be

$$
q^{\prime \prime \prime}= \begin{cases}Q_{0}\left(T-T_{C}\right), & \text { for } T \geq T_{C} \\ 0, & \text { for } T<T_{C}\end{cases}
$$

where $Q_{0}$ is a heat generation constant which may be either positive or negative. This source term represents the heat generation when $Q_{0}>0$ and the heat absorption when $Q_{0}<0$.

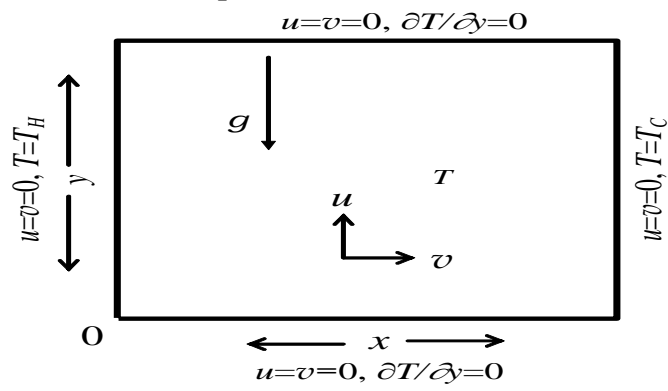

Fig.1: Physical model and coordinate system. 
We further assume unsteady two-dimensional laminar free convective flow of viscous incompressible fluid having constant properties. The effect of buoyancy is included through the well-known Boussinesq approximation. Under the above assumption, the equations for mass continuity, momentum and energy take the following form:

$$
\begin{aligned}
& \frac{\partial u}{\partial x}+\frac{\partial v}{\partial y}=0, \\
& \frac{\partial u}{\partial t}+u \frac{\partial u}{\partial x}+v \frac{\partial u}{\partial y}=-\frac{1}{\rho} \frac{\partial p}{\partial x}+v\left(\frac{\partial^{2} u}{\partial x^{2}}+\frac{\partial^{2} u}{\partial y^{2}}\right) \\
& \frac{\partial v}{\partial t}+u \frac{\partial v}{\partial x}+v \frac{\partial v}{\partial y}=-\frac{1}{\rho} \frac{\partial p}{\partial y}+v\left(\frac{\partial^{2} v}{\partial x^{2}}+\frac{\partial^{2} v}{\partial y^{2}}\right)+\rho g \beta\left(T-T_{C}\right) \\
& \frac{\partial T}{\partial t}+u \frac{\partial T}{\partial x}+v \frac{\partial T}{\partial y}=\alpha\left(\frac{\partial^{2} T}{\partial x^{2}}+\frac{\partial^{2} T}{\partial y^{2}}\right)+\frac{Q_{0}}{\rho C_{p}}\left(T-T_{C}\right)
\end{aligned}
$$

where $(u, v)$ are the velocity components along the $(x, y)$ axes, $g$ is the acceleration due to gravity, $\rho$ is the fluid density, $\alpha$ is the thermal diffusivity, $\beta$ is the coefficient of thermal expansion, $v$ is the kinematic viscosity of the fluid, $C_{p}$ is the specific heat at constant pressure.

To make the above equations dimensionless, we introduce the following non-dimensional variables

$$
\begin{aligned}
& X=\frac{x}{H}, Y=\frac{y}{H}, \quad \tau=\frac{t}{H^{2} / v}, \quad U=\frac{u}{v / H}, \\
& V=\frac{v}{v / H}, \quad P=\frac{p}{\rho v^{2} / H^{2}}, \quad \theta=\frac{T-T_{C}}{T_{H}-T_{C}}
\end{aligned}
$$

where $v(=\mu / \rho)$ is the reference kinematic viscosity and $\theta$ is the non-dimensional temperature.

Substitution of the dimensionless variables (6) into equations (2)-(5) lead to the following equations:

$$
\begin{aligned}
& \frac{\partial \Omega}{\partial \tau}+\frac{\partial(U \Omega)}{\partial X}+\frac{\partial(V \Omega)}{\partial Y}=\left(\frac{\partial^{2}}{\partial X^{2}}+\frac{\partial^{2}}{\partial Y^{2}}\right) \Omega+\frac{R a}{\operatorname{Pr}} \frac{\partial \theta}{\partial X}, \\
& \frac{\partial \theta}{\partial \tau}+\frac{\partial(U \theta)}{\partial X}+\frac{\partial(V \theta)}{\partial Y}=\frac{1}{\operatorname{Pr}}\left(\frac{\partial^{2}}{\partial X^{2}}+\frac{\partial^{2}}{\partial Y^{2}}\right) \theta+\lambda \theta,
\end{aligned}
$$

where

$$
\Omega=-\left(\frac{\partial^{2}}{\partial X^{2}}+\frac{\partial^{2}}{\partial Y^{2}}\right) \psi
$$

is the vorticity function and $\psi$ is the stream function defined by :

$$
U=\frac{\partial \psi}{\partial Y}, \quad V=-\frac{\partial \psi}{\partial X} .
$$

In the above equations $R a$ is the Rayleigh number, $\operatorname{Pr}$ is the Prandtl number and $\lambda$ is the heat generation parameter defined respectively by the following relations.

$$
R a=\frac{\beta\left(T_{H}-T_{C}\right) H^{3}}{\alpha v}, \quad \operatorname{Pr}=\frac{v}{\alpha} \quad \text { and } \lambda=\frac{Q_{0} H^{2}}{\mu C_{p}}
$$

The dimensionless initial and boundary conditions are:

$$
\begin{array}{ll}
U=V=\theta=0 & \text { for } \tau=0 \\
U=V=0, \quad \theta=1 & \text { for } 0 \leq Y \leq 1 \text { at } X=0 \\
U=V=0, \quad \theta=0 & \text { for } 0 \leq Y \leq 1 \text { at } X=1 \\
U=V=0, \quad \frac{\partial \theta}{\partial Y}=0 & \text { for } 0 \leq X \leq 1 \text { at } Y=0 \\
U=V=0, \quad \frac{\partial \theta}{\partial Y}=0 & \text { for } 0 \leq X \leq 1 \text { at } Y=1
\end{array}
$$


Once we know the numerical values of the temperature function we may obtain the rate of heat transfer in terms of the local Nusselt number, $N u$ from the heated portion of the bottom wall using the following relation:

$$
N u=-\left(\frac{\partial T}{\partial X}\right)_{X=0}
$$

The average Nusselt number, $N u_{a v}$ is given by

$$
N u_{a v}=-\int_{0}^{1}\left(\frac{\partial T}{\partial X}\right)_{X=0} d Y
$$

\section{Numerical Procedure}

The governing equations along with the boundary conditions are solved numerically, employing finite difference method. The energy equation is solved using explicit upwind finite difference technique and the stream function equation is solved by SOR (successive over relaxation) scheme. To speed up the convergence, the over relaxation parameter for the stream function solutions, is not fixed for all mesh sizes. A semi-dynamic model has been used for calculating over relaxation parameter for every orientation of grid size(see Roache [21]). For example, the values of the over relaxation parameter for $41 \times 41$ and $51 \times 5$ grid sizes are not same. The buoyancy and diffusive terms are discretised by using central differencing while the use of upwind differencing is preferred for convective terms for numerical stability. By using the initial and boundary conditions, the discretised transient equations are then solved explicitly by marching in time until an asymptotic steady state solution is reached. Convergence of iterations for stream function solution is obtained at each time step. The following criterion is employed to check for steady state solution

$$
\sum_{i, j}\left|\Phi_{i, j}^{n+1}-\Phi_{i, j}^{n}\right| \leq 10^{-5}
$$

Where $\Phi$ stands for $\psi, \Omega$ and $\theta ; n$ refers to time and $i$ and $j$ refer to space coordinates. The time step $\Delta \tau$ used in computations is varied between 0.00005 and 0.00001 depending on the Rayleigh numbers.

The aspect ratio considered is unity and the value of $\Delta X=\Delta Y=1 /(N-1), N$ is the number of grid points in the $Y$ direction. A grid independence study is conducted using four different uniform grid sizes $31 \times 31,41 \times 41$, $51 \times 51$ and $61 \times 61$ and it is observed that a further refinement of grid of $51 \times 51$ and $61 \times 61$ does not have a significant change on the results of the maximum stream function and the average Nusselt number. For computational economy, a $51 \times 51$ mesh has been used throughout for this simulations described below.

Table 1: Comparisons of present numerical results with the benchmark solutions (listed in bracket) [2] for the

\begin{tabular}{|l|l|l|l|l|}
\hline \multicolumn{5}{|l|}{ case $\operatorname{Pr}=0.71, \lambda=0.0$ and $51 \times 51$ meshes } \\
\hline $\mathrm{Ra}$ & $\left|\psi_{\max }\right|$ & $\mathrm{U}_{\max }$ & $\mathrm{V}_{\max }$ & $N u_{\mathrm{av}}$ \\
\hline $10^{3}$ & 1.1655 & $3.616(3.649)$ & $3.665(3.697)$ & $1.1298(1.118)$ \\
\hline $10^{4}$ & 5.1283 & $16.230(16.178)$ & $19.468(19.617)$ & $2.265(2.243)$ \\
\hline $10^{5}$ & $10.141(9.612)$ & $34.92(34.73)$ & $68.43(68.59)$ & $4.653(4.519)$ \\
\hline
\end{tabular}

\section{Results and Discussion}

Numerical results are presented in order to determine the effects of internal heat generation and the Rayleigh number in presence of heat generation flow in a square cavity. Values of the heat generation parameter $\lambda$, between 0.0 and 20.0 and the Rayleigh number $R a\left(=10^{3}, 10^{4}, 10^{5}, 10^{6}\right)$ are considered here.

\subsection{Benchmarking}

The corresponding problem without the effect of heat generation has been investigated by de Vahl Davis [2] for different values of the Rayleigh number for $\operatorname{Pr}=0.71$. With $51 \times 51$ mesh for the case of $\operatorname{Pr}=0.71$ and $\lambda=0.0$, the comparison of the maximum values of $\psi, U, V$ and average Nusselt number, $N u_{\mathrm{av}}$ are presented in the following Table 1, which shows a good agreement of the present solutions with those of de Val Devis [2].

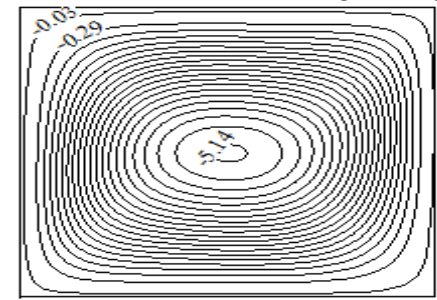

$\lambda=0.0, \psi_{\max }=0.0$

$\psi_{\min }=-5.1724$

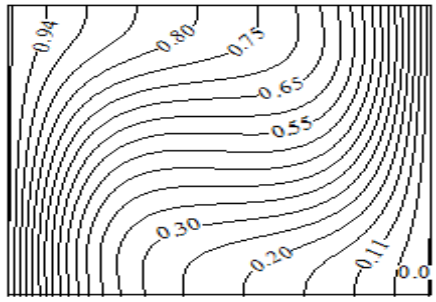

$\lambda=0.0, \theta_{\max }=1.0$ 

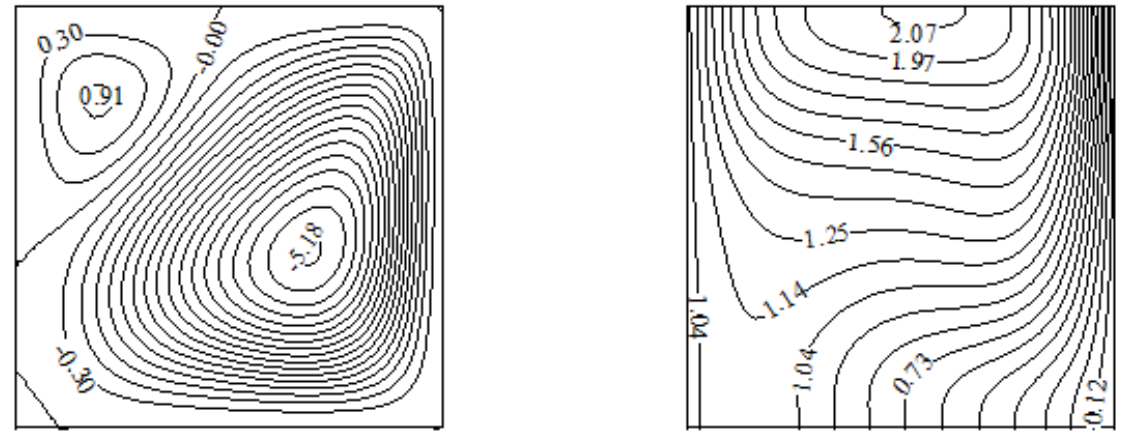

$\lambda=10.0, \psi_{\max }=0.9545$,

$\psi_{\min }=-5.2185$

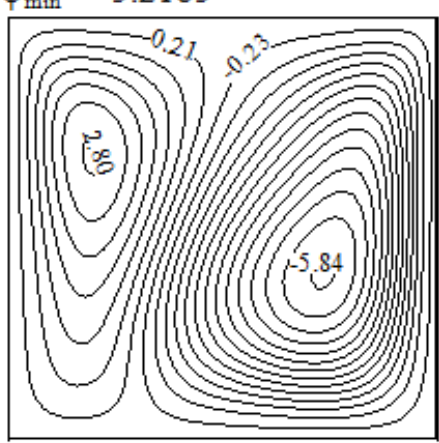

$\lambda=10.0, \theta_{\max }=2.0824$

$\lambda=15.0, \psi_{\max }=2.8549$,

$\psi_{\min }=-5.8997$

$\psi_{\min }=-5.8997$

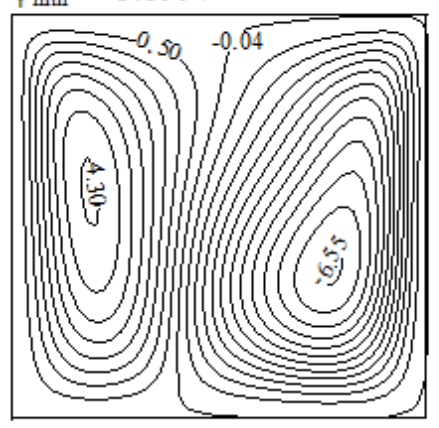

$\lambda=20.0, \psi_{\max }=4.3752$,

$\psi_{\min }=-6.6278$

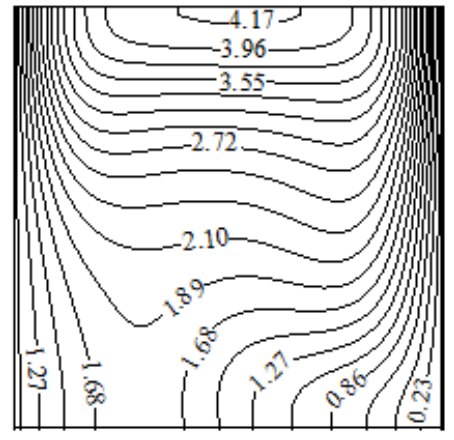

$\lambda=15.0, \theta_{\max }=4.1941$

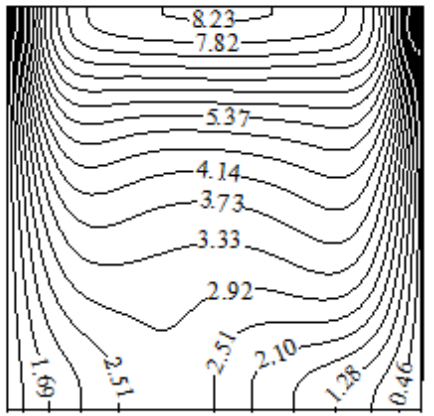

$\lambda=20.0, \theta_{\max }=8.2858$

Fig. 2: Streamlines and isotherms for $\mathrm{Ra}=10^{4}$

\subsection{Effect of varying the heat generation parameter on the flow field and heat transfer}

We consider first the effect of internal heat generation on the flow field. The resulting flows and temperature distributions are depicted in Fig. 2. In Fig. 2 (top) we present the streamlines for increasing values of the heat generation parameter, $\lambda(=0.0,10.0,15.0,20.0)$ while $R a=10^{4}$. In these figures, without heat generation, there is only one cell, called primary cell has been seen. With the effect of heat generation, a secondary cell has been developed in the top-left corner of the cavity. The increasing rate of heat generation leads to increase the flow rate in the secondary cell as well as increase in its size until it occupies almost half of the total cavity. This effect of internal heat generation on the flow field is reasonable since internal heat generation assists buoyancy forces by accelerating the fluid flow. On the other hand, the fluid temperature increases significantly due to effect of heat generation which is shown in isotherms in Fig. 2 (bottom). It is clearly seen that, owing to increase the heat generation, the fluid temperature exceeds the surface temperature that negates the heat transfer from the heated surface which is also illustrated in Fig. 4 (a).

Fig 3 shows the effect of Rayleigh number on the flow and temperature field where the internal heat generation is fixed at $\lambda=10.0$. For the lower Raleigh number two convective cells dominate the flow, however, with increase of Rayleigh number the primary cell becomes larger and occupies whole domain when Ra $=10^{6}$. This behaviour is almost opposite of the effect of heat generation from lower to higher for a fixed Raleigh number (see in Fig 2). As we discuss earlier that the internal heat generation accelerates the flow and eventually it 
has an influence on buoyancy, however, for a fixed $\lambda$ when Raleigh number increases, the buoyancy effect accelerates and dominates the flow, although there is a weak internal heat generation influence on the flow filed. The same phenomenon has been seen in the temperature contours in Fig 3(bottom). Initially, when the Raleigh number is small the internal fluid temperature is higher but it decreases with increase of Ra. For $\mathrm{Ra}=10^{6}$ the flow is stratified which is similar to a side heated cavity flow with no internal heat generation.

\subsection{Effect of heat generation and the Rayleigh number on the flow field and heat transfer}

Fig. 4 (a) shows how the presence of heat generation inside the fluid influences the overall predictions of heat transfer. Initially when $\lambda=0$, the heat transfer on the hot surface is positive, however, as the amount of heat generation increases, the total heat transfer decreases and becomes negative. This is due to the fact that the heat generation mechanism creates a layer of hot fluid near the hot surface and at some level when $\lambda$ is larger, the resultant temperature of fluid exceeds the surface temperature. The rate of heat transfer also decreases with the height of the cavity. When there is no heat generation effect, the rate is uniform, however, when $\lambda=20.0$ the temperature decreasing largely near the top of the surface line. Fig 4(b) shows the effect of heat transfer from the hot surface on Rayleigh number when the heat generation parameter is fixed at $\lambda=10.0$. For smaller Rayleigh numbers, the heat transfer rate is negative $\left(\mathrm{Ra}=10^{3}, 10^{4}\right)$. That means this surface is loosing the heat whereas, for higher Rayleigh number e.g. $\mathrm{Ra}=10^{6}$, heat transfer rate is positive, although it is decreasing with height of the surface again. For higher values of Ra, there is a strong effect of buoyancy force in the flow field which causes to circulate the internally generated heat throughout the cavity. Hence the hot layer, which has formed near the hot surface, disappears and the heat from the hot surface defuses in the cavity. In Fig. 5, we have depicted the average rate of heat transfer from the heated wall in terms of the average Nusselt number, $N u_{\mathrm{av}}$ for different values of the heat generation parameter $\lambda$ against the Rayleigh number $\operatorname{Ra}$ ( log-scale has been used for Ra ). It is seen that for large values of $\lambda$ the average Nusselt number $N u_{\mathrm{av}}$ decreases and for large values of Ra, the average rate of heat transfer increases, which is expected according to Fig. 4.

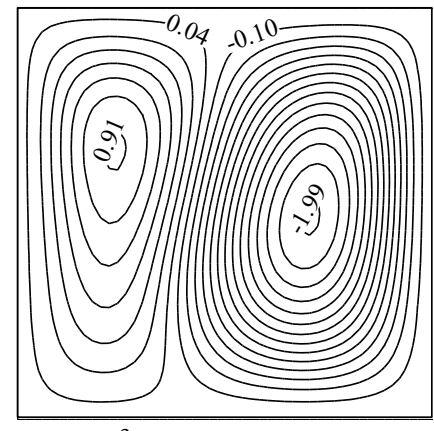

$\mathrm{Ra}=10^{3}, \psi_{\max }=0.9324$,

$\psi_{\min }=-2.0073$

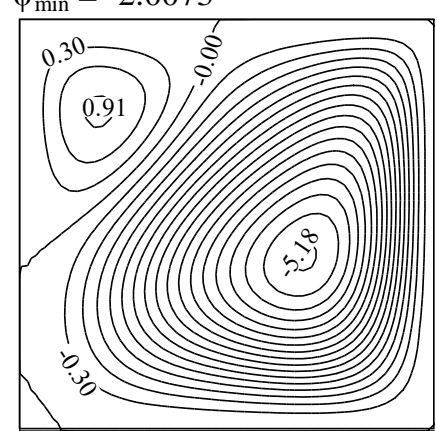

$\mathrm{Ra}=10^{4}, \psi_{\max }=0.9545$,

$\psi_{\min }=-5.2185$

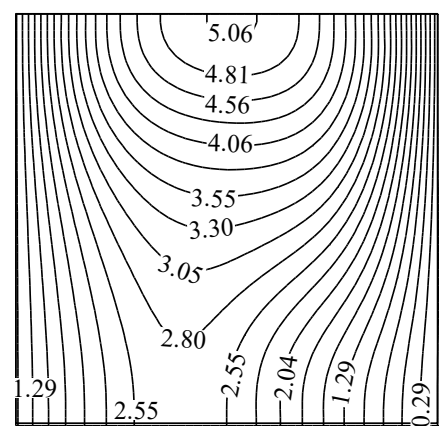

$\mathrm{Ra}=10^{3}, \theta_{\max }=5.0942$

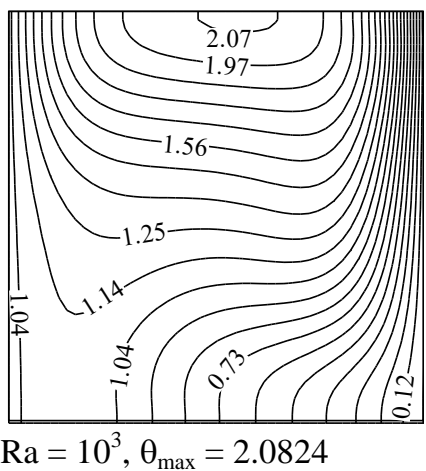




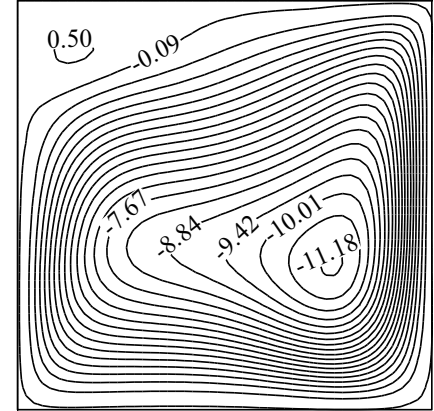

$\mathrm{Ra}=10^{5}, \psi_{\max }=0.5761$,

$\psi_{\min }=-11.2534$

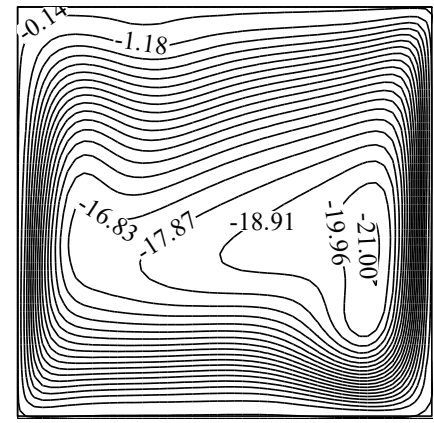

$\mathrm{Ra}=10^{6}, \psi_{\max }=0.0$, $\psi_{\min }=-21.1405$

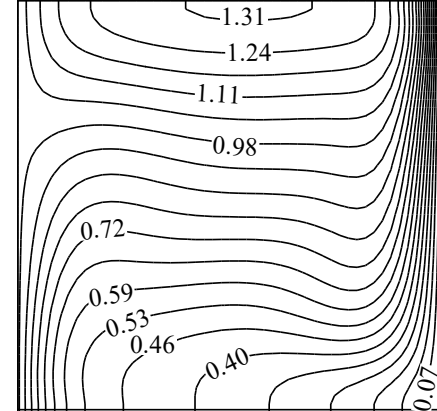

$\mathrm{Ra}=10^{5}, \theta_{\max }=1.3163$

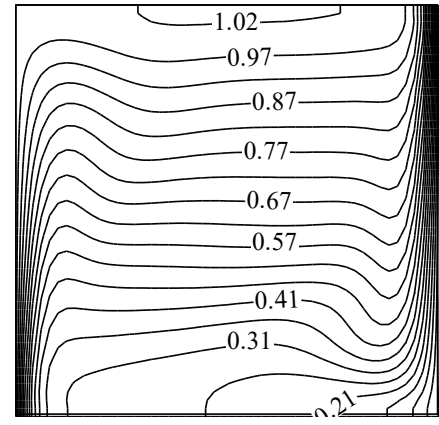

$\mathrm{Ra}=10^{6}, \theta_{\max }=1.0306$

Fig. 3: Streamlines and isotherms for $\lambda=10$
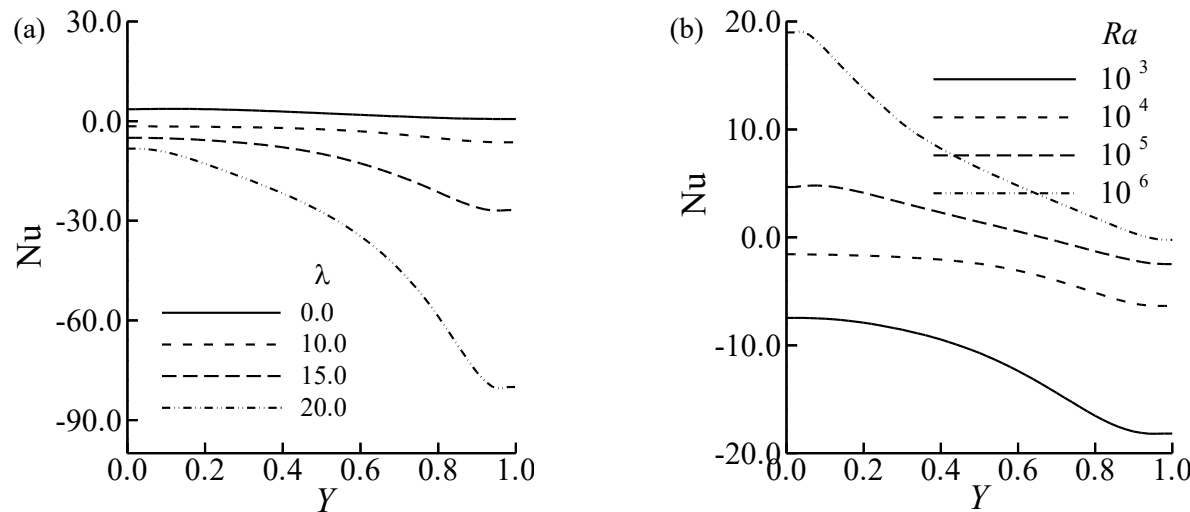

Fig. 4: Rate of heat transfer from the hot wall (a) varying $\lambda$ with $R a=10^{4}$ and (b) varying $R a$ with $\lambda=10$.

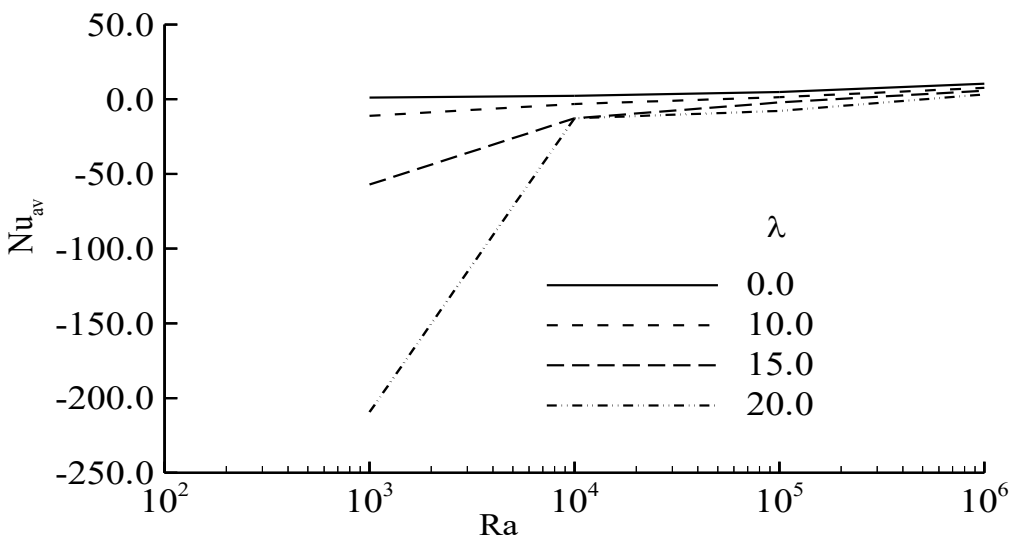

Fig. 5: Average Nusselt number from the heated wall for varying $\lambda$ against $R a$. 


\section{Conclusions}

In the present paper a problem on natural convection laminar flow in a side heated square cavity with internal heat generation has been investigated numerically by employing an upwind finite difference method together with a Successive Over-Relaxation (SOR) technique. The results have been presented for the chosen fluid of Prandtl number $\operatorname{Pr}=1.0$, heat generation parameter $\lambda(=0.0,10.0,15.0,20.0)$ and the Rayleigh number $\operatorname{Ra}(=$ $10^{3}, 10^{4}, 10^{5}, 10^{6}$ ). From the present investigation the following conclusion may be drawn:

- Increase in the values of heat generation parameter leads to develop a secondary cell on the left upper corner of the cavity and increases the flow rate in the secondary cell as well as increase its size until it occupies almost half of the cavity.

- The temperature of the fluid in the cavity also increases due to increase the internal heat generation and hence the local and average rate of heat transfer from the left wall decrease.

- For increasing values of the Rayleigh number, the flow rate increases in both clock wise and anti-clockwise direction.

- The local and average rate of heat transfer from the heated wall increase owing to increase the Rayleigh number.

\section{Reference}

[1] S. Ostrach, Natural convection in enclosures. ASME J. Heat Transfer 110 (1988) 1175-1190.

[2] G. De Vahl Davis, Natural convection of air in a square cavity, A bench mark numerical solution. Int. J. Numer. Methods Fluids 3 (1983) 249-264

[3] A. Valincia, R. L. Frederick, Heat transfer in square cavities with partially active vertical walls. Int. J. Heat Mass Transfer 32 (1989) 1567-1574.

[4] E. E. Selamet, V. S. Arpaci, C. Borgnakke, Simulation of laminar buoyancy driven flows in an enclosure. Numerical Heat Transfer 22 (1992) 401-420.

[5] M. Hasnaoui, E. Bilgen, P. Vasseure, Natural convection heat transfer in rectangular cavities heated from below. J. Thermophysics. Heat Transfer 6 (1995) 255-264.

[6] L. G. Sundstrom, S. Kimura, On laminar free convection in inclined rectangular enclosure. J. Fluid Mech. 313 (1996) $343-366$

[7] M. November, M. W. Nansteel, Natural convection in rectangular enclosures heated form below and cooled along one side. Int. J. Heat Mass Transfer 30 (1986) 2433-2440

[8] M. M. Ganzarolli, L. F. Milanez, Natural convection rectangular enclosures heated from below and symmetrical cooled from the sides. Int. J. Heat mass Transfer 38 (1995) 1063-1073.

[9] O. Aydin, J. Yang, Natural convection in enclosures with localized heating from below and symmetrical cooling from sides. Int. J. Numer. Methods Heat Fluid Flow 10 (2000) 518-529.

[10] O. Aydin, A. T. Unal, Natural convection in rectangular enclosures heated from one side and cooled from the ceiling. Int. J. Heat Mass Transfer 42 (1999) 2345-2355.

[11] M. A. R. Sharif, T. R. Mohammad, Natural convection in cavities with constant flux heating at the bottom wall and isothermal cooling from the sidewalls. Int. J. Thermal Sci. 44 (2005) 865-878.

[12] I. Sezai, A. A. Mohammad, Natural convection from a discrete heat source on the bottom of a horizontal enclosure. Int. J. Heat Mass Transfer 43 (2000) 2257-2266.

[13] H. N. Dixit, V. Babu, Simulation of high Rayleigh number natural convection in a square cavity using the lattice Boltzmann method. Int. J. Heat Mass Transfer 49 (2006) 727-749.

[14] G. S. Barozzi, M. A. Corticelli, Natural convection in cavities containing internal sources, Heat Mass Transfer 36 (2000) $473-480$

[15] Z. G. Du, E. Bilgen, Natural convection in vertical cavities with internal heat generation porous medium, Warm- und Stoffubertragung 27 (1992) 149-155.

[16] A. C. Baytas, Buoyancy-driven flow in an enclosure containing time periodic I nternal heat sources, Heat Mass Transnfer 31 (1996) 113-119.

[17] K. Vajravelu, A. Hadjinicolaou, Heat transfer in a viscous fluid over a stretching sheet with viscous dissipation and internal heat generation. Int. Comm. Heat Mass Transfer 20 (1993) 417-430.

[18] M. M. Molla, M. A. Hossain, L. S. Yao, Natural convection flow along a vertical wavy surface with heat generation/absorption. Int. J. Thermal Sci. 43 (2004) 157-163.

[19] M. M. Molla, M. A. Hossain, M. C. Paul, Natural convection flow from an isothermal circular cylinder in presence of heat generation. Int. J. Engn. Sci. 44 (2006) 949-958.

[20] M. M. Molla, M. A. Hossain, M. A. Taher, Magnetohydrodynamic natural convection flow on a sphere with uniform heat flux in presence of heat generation. Acta Mechanica (in press).

[21] P. J. Roache, Computational Fluid Dynamics, Hermosa, Albuquerque. NM, 1982. 